\title{
Through a womb, darkly: Methodological problems in a recent study of fetal visual perception
}

\author{
Anne M. Scheel ${ }^{1,}{ }^{*}$, Stuart J. Ritchie ${ }^{2}$, Nicholas J. L. Brown ${ }^{3}$, and Steven L. Jacques ${ }^{4,5,6}$ \\ ${ }^{1}$ School of Innovation Sciences, Eindhoven University of Technology, Eindhoven, The Netherlands \\ ${ }^{2}$ Centre for Cognitive Ageing and Cognitive Epidemiology, The University of Edinburgh, Edinburgh, UK \\ ${ }^{3}$ University Medical Center, University of Groningen, Groningen, The Netherlands \\ ${ }^{4}$ Department of Biomedical Engineering, Tufts University, Medford, MA, USA \\ ${ }^{5}$ Department of Biomedical Engineering, Oregon Health Science University, Portland, OR, USA \\ ${ }^{6}$ Department of Dermatology, Oregon Health Science University, Portland, OR, USA \\ *Correspondence: a.m.scheel@tue.nl
}

Unreviewed preprint, October 2017

\section{Summary}

Reid et al. [1] analyzed data from 39 third-trimester fetuses, concluding that they showed a preferential head-orienting reaction towards lights projected through the uterine wall in a face-like arrangement, as opposed to an inverted triangle of dots. These results imply not only that assessment of visual-perceptive responses is possible in prenatal subjects, but also that a measurable preference for faces exists before birth. However, we have identified three substantial problems with Reid et al.'s method and analyses, which we outline here.

\section{Main Text}

First, a number of analytical decisions render the results unclear. Unlike previous paradigms in the newborn literature, which involve degree of head turning [2] or preferential orienting towards simultaneously presented stimuli [3], Reid et al. [1] measured orienting responses to a single stimulus (face-like or inverted). Also unlike prior studies [4], Reid et al. did not analyze orienting responses per trial, instead aggregating across all five trials per condition per subject. The resulting score is difficult to interpret: It counts a fetus with repeated head motion to and from the stimulus as showing a greater preference than one making a single movement and then remaining fixated on the stimulus for the entire trial. Moreover, a value of (for example) 4 could mean one head turn in $4 / 5$ trials, or 4 turns in 1/5 trials. It is thus not clear that more head turns indicate greater preference for the stimulus. A more easily-interpretable measure is the occurrence of any head movement toward the stimulus for each fetus, regardless of frequency. We performed this analysis ( $\mathrm{R}$ code available at https://osf.io/ctqxw/), finding that 20 subjects turned their head towards the upright 
(face-like) stimulus but not toward the inverted stimulus, compared to 19 who oriented towards both, neither, or only towards the inverted stimulus. Even considering number of head turns as an ordinal variable, we find that only 21 of 39 fetuses (53.8\%) turned their head more often towards the upright than the inverted stimulus. These numbers do not provide compelling support for the face-preference hypothesis. We made a request to obtain the trial-level raw data for reanalysis, but Dr. Reid (personal communication, $15^{\text {th }}$ June, 2017) informed us that he was unable to share these as it would require the coding within the Observer software system to be repeated.

Second, the light levels may have been insufficient for many fetuses to perceive the stimuli. Reid et al. described using lasers of three different power levels for three different ranges of tissue thickness, estimating intrauterine illuminance of 16-36 lx for these values. However, using equation 2 from ref. [5] and the actual maternal tissue thickness for each subject, we calculate that 10 fetuses had intrauterine illuminances below the 10-lx threshold suggested as the minimum for fetal vision [5] (available in our R code). Further, the authors did not code whether fetuses had their eyes open, a measure that three of them recommended in an earlier review [6, p. 46]. One of the authors had previously reported that 32- to 36-week-old fetuses opened their eyes 1.88-6.50 times per 10 minutes [7, Table 9.2]. Assuming a total presentation duration of 50 seconds ( 5 seconds $\times 5$ trials $\times 2$ stimuli), the probability of any single fetus opening its eyes even just once during the whole experiment is between 0.16 and 0.54. It therefore seems unlikely that more than half of the fetuses were able to view and react to both stimuli. This could have been avoided by including a control condition with non-task-relevant stimuli or by recording whether or not the fetuses had their eyes open.

Third, even for fetuses that were able to see the stimuli, distinguishing the "upright" and "inverted" versions was likely barely possible. Reid et al.'s formula to calculate light scattering in the maternal tissue (see caption to their Figure 1) seems to assume that a point light source is scattered once by a fixed angle on arriving at the mother's skin surface, spreading out evenly to form a cone with the diameter of the circles shown in Reid et al.'s Figures 1c and $1 \mathrm{~d}$.

However, in tissue of non-trivial thickness, scattering takes place repeatedly via an essentially stochastic process (depending on the anisotropy of the tissue, g). Monte Carlo simulations of the shapes visible to the fetus at a tissue thickness of $26.4 \mathrm{~mm}$ (median tissue thickness in the full sample) are shown in Figure 1. The resulting upright shape (Figure 1c) does not approximate a human face and is difficult to distinguish from the inverted one (Figure 1d). The median tissue thickness for subjects who turned towards the upright facial pattern at least once was $27.4 \mathrm{~mm}$; one third of the sample (13 mothers) had a tissue thickness above $30 \mathrm{~mm}$. Thus, it appears unlikely that many of the fetuses could have distinguished the two types of stimuli.

Reid et al. raised an intriguing question about prenatal visual perception and presented a first step to investigate it with an interesting new experimental approach. This letter prompts a consideration of their method, and of how well a face-like image would penetrate to the fetus. We conclude that there are three main analytical and experimental problems: the lack 

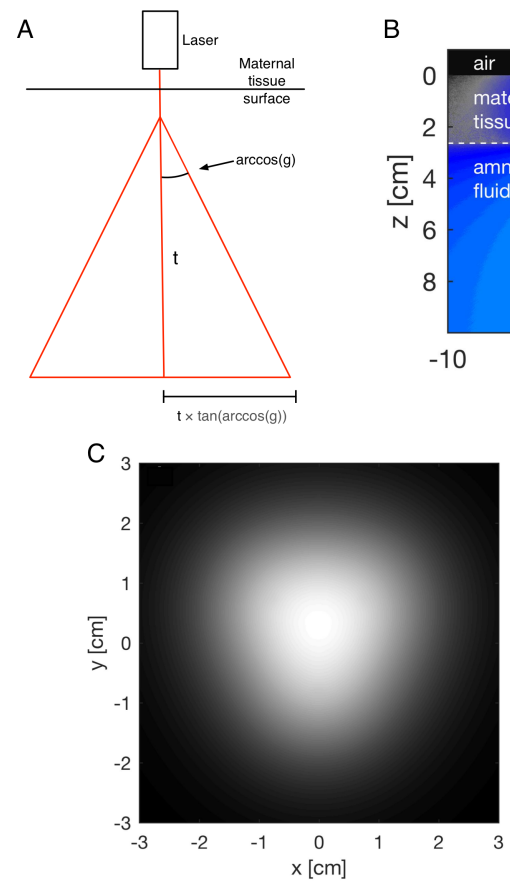

B

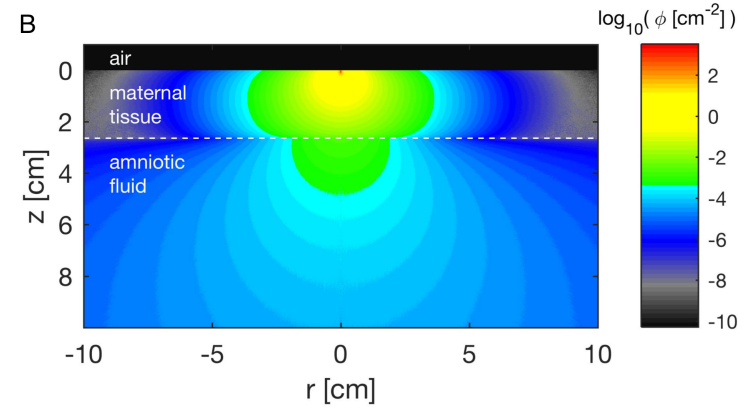

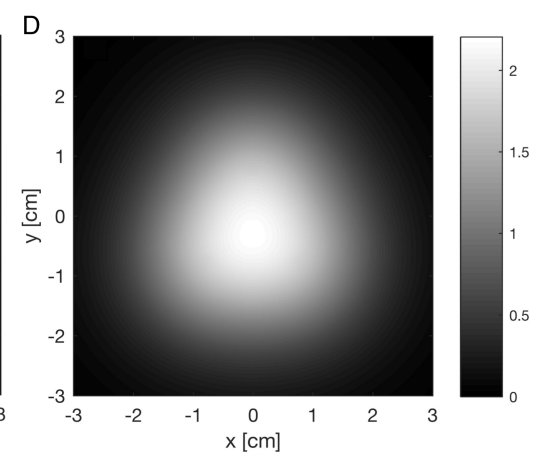

Figure 1. A. Cone of light produced by a laser beam hitting the mother's skin and being scattered inside the abdominal tissue, according to Reid et al.'s model. $t$ is the thickness of the uterine tissue. Where $t=30 \mathrm{~mm}$, the radius of the base of the cone is $6 \mathrm{~mm}$, meaning that the fetus allegedly saw a disk of $12 \mathrm{~mm}$ diameter. $B$. Light distribution within the abdominal tissue and amniotic fluid for $t=26.4 \mathrm{~mm}$ (median tissue thickness), estimated with Monte Carlo simulations. Tissue parameters: blood volume fraction $(B)=0.005$, oxygen saturation $(S)=0.75$, water content $(W)=0.65$, absorption coefficient $\left(\mu_{\mathrm{a}}\right)=0.0850 \mathrm{~cm}^{-1}$, scattering coefficient $\left(\mu_{\mathrm{s}}\right)=100 \mathrm{~cm}^{-1}$, anisotropy $(g)=0.90$, refractive index $(n)=1.4$; amniotic fluid parameters: $B=0, S=0, W=0.95, \mu_{\mathrm{a}}=0.0030 \mathrm{~cm}^{-1}$, $\mu_{s}=100 \mathrm{~cm}^{-1}, g=0.99, \mathrm{n}=1.35$. C. and D. Estimated light pattern visible to the fetus at $t=26.4 \mathrm{~mm}$ in the upright (face-like) and inverted conditions, respectively. Note: C. and D. display the estimated fluence rate $\left(W / \mathrm{cm}^{2} \mathrm{per}\right.$ $W$ delivered) in the $x-y$ plane at depth $z=26.4 \mathrm{~mm}$ (dashed white line in B.).

of per-trial data means that the dependent variables do not present a valid measure of visual preference, and re-analyses show that only around half of the subjects exhibited the effect; most of the fetuses might not have been able to perceive the stimuli; and even for those that could, the upright shape may not have appeared face-like or distinguishable from the inverted shape. We conclude that the study cannot convincingly answer the question of whether fetuses have a preference for face-like visual stimuli.

\section{Acknowledgements}

We thank Vincent Reid for his helpful responses to our enquiries about the study, and Malte Elson for his comments on an early draft of this comment. 


\section{References}

1. Reid, V. M., Dunn, K., Young, R. J., Amu, J., Donovan, T., and Reissland, N. (2017). The human fetus preferentially engages with face-like visual stimuli. Curr. Biol. 27, 1825-1828.e3. doi: 10.1016/j.cub.2017.05.044

2. Johnson, M. H., Dziurawiec, S., Ellis, H., and Morton, J. (1991). Newborns' preferential tracking of face-like stimuli and its subsequent decline. Cognition 40, 1-19. doi: 10.1016/0010-0277(91)90045-6

3. Valenza, E., Simion, F., Macchi Cassia, V., and Umiltà, C. (1996). Face preference at birth. J. Exp. Psychol. Hum. Percept. Perform. 22, 892-903. doi: 10.1037/0096-1523.22.4.892

4. Simion, F., Valenza, E., Umiltà, C., and Dalla Barba, B. (1998). Preferential orienting to faces in newborns: a temporal-nasal asymmetry. J. Exp. Psychol. Hum. Percept. Perform. 24, 1399-1405. doi: 10.1037/0096-1523.24.5.1399

5. Del Giudice, M. (2011). Alone in the dark? Modeling the conditions for visual experience in human fetuses. Dev. Psychobiol. 53, 214-219. doi: 10.1002/dev.20506

6. Dunn, K., Reissland, N., and Reid, V. M. (2015). The functional foetal brain: A systematic preview of methodological factors in reporting foetal visual and auditory capacity. Dev. Cogn. Neurosci. 13, 43-52. doi: 10.1016/j.dcn.2015.04.002

7. Reissland, N., Francis, B., and Buttanshaw, L. (2015). The fetal observable movement system (FOMS). In Advancing Research in Fetal Development, N. Reissland \& B. S. Kisilevsky, eds. (New York: Springer), p. 153. doi: 10.1007/978-3-319-22023-9_9 